\title{
Microanalysis of Coolant Mists Generated from Wet Cutting of Art Glass
}

\author{
R.B. Simmons \\ Georgia State University, Biology Dept., Atlanta, GA 30303 \\ Briarwillow, LLC; Atlanta, GA 30345
}

Typically glass is cut by a 'score and break' process in which the surface of the glass is marked by a diamond or hardened metal device. This marking process sets up internal stresses within the body of the glass and when opposing pressure is applied to the score area the glass will separate along this line. This process is common in both industrial and hobby applications where straight lines or gentle curves are required. So-called cold working processes applied to glass in both the industrial and art glass areas often involve grinding, polishing or cutting using more aggressive mechanical methods. The use of water, propylene glycol or mineral oil as cooling and lubricating fluids for these processes is common. As art glass, including stained glass and hot glass (blowing, kiln forming or flame working), has become more consumer friendly an increase in small scale commercial and hobby-level cold working applications has followed. Many of the artists involved in these operations are unaware of the potential for exposure to hazardous materials generated in cold working processes. This paper examines the physical and chemical exposure hazards from 'wet cutting' glass materials.

Wet-cutting of glass is most often accomplished using steel saw blades coated or embedded with industrial diamond particles. The size of the diamond particles is determined by the specific application, rough cutting using larger particles while fine cutting requires both smaller diamond particles and generally thinner kerf blades. Blades may be round steel discs similar to common saw blades with diamond particles fixed along a smooth rim, diamond coated bands for band saws or diamond coated wire for so-called 'ring' saws. Water is the most commonly used cooling agent and lubricant for these saws, though propylene glycol or oils may also be used. During the cutting operation the diamond particles abrade the glass. The lubricating fluid captures much of the abraded material and carries it to a reservoir as it also cools the cutting surface. All of these operations generate a mist of coolant that is ejected into the atmosphere around the cutting area and may be spread over a wide area. This mist contains small particles of glass and may present hazards to the operator of these saws.

Scanning electron microscopy (SEM) has shown that glass particles contained in the mist range in size from tens of micrometers to well under 1 micrometer in diameter and display sharp points and edges (Fig 1, A-D). Small aerosolized particles may be inhaled and taken deep into both the upper and lower respiratory tracts. These present a hazard for lung damage such as that seen in mine workers with prolonged exposure resulting in airway irritation and possible fibrosis of the lungs (silicosis). Metals are often used as coloring agents in glass and can be detected by Energy Dispersive X-ray microanalysis. These materials may present a localized toxicity hazard if the particles become embedded in lung, nasal or eye area mucosal tissues.

Workers using wet cutting machines on the industrial level are generally required to wear appropriate protective equipment. Smaller scale commercial workers and hobbyists are often unaware of the respiratory hazards associated with wet glass cutting and may not use safety equipment such as face shields and air filtering devices for respiratory protection. Anyone using wet 
cutting or grinding equipment should familiarize themselves with all hazards associated with it and take appropriate measures to prevent or minimize exposure to these particles.
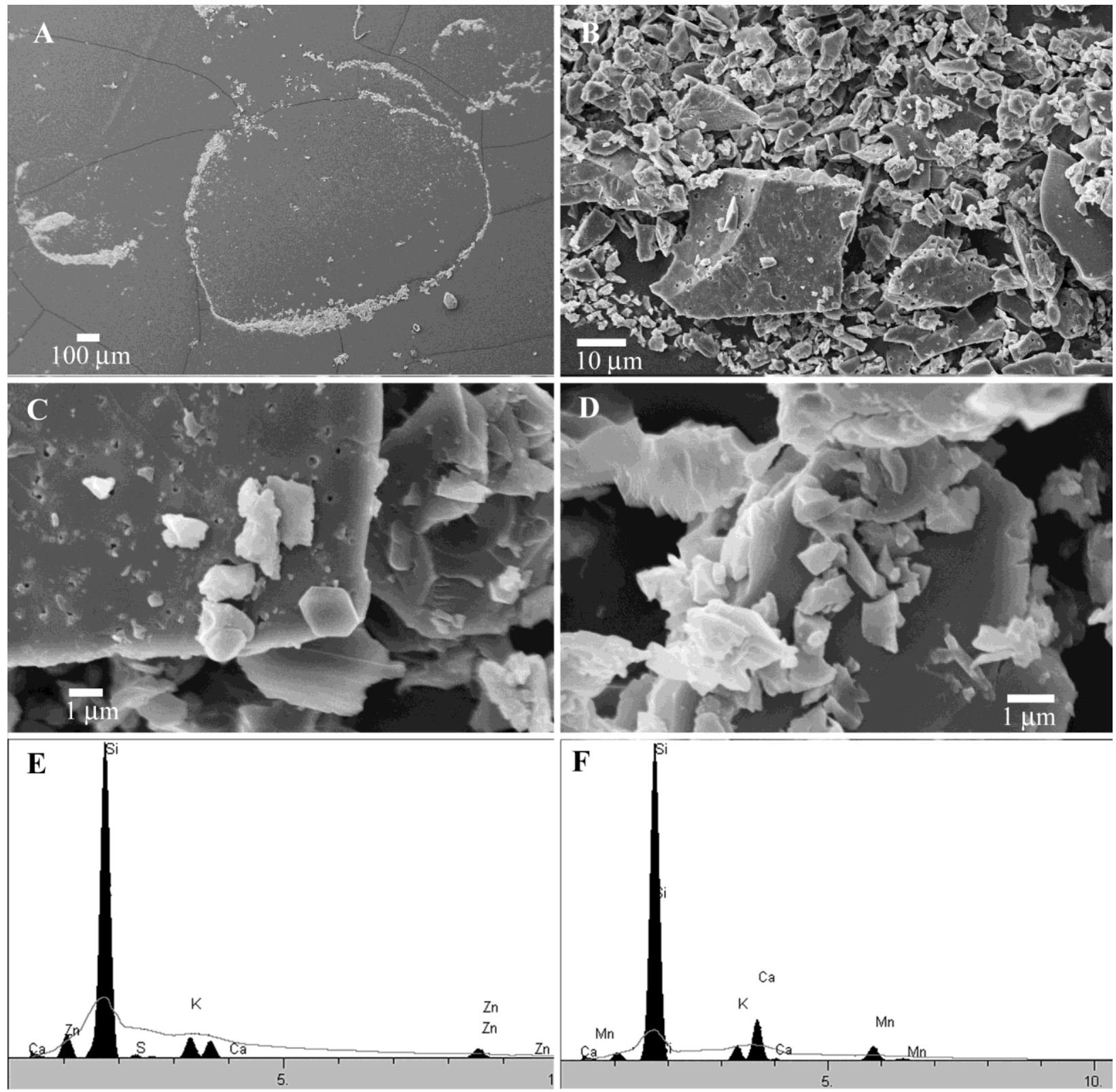

Figure 1. A. Dried mist droplet showing deposition of particles on the substrate. Bar $=100 \mu \mathrm{m}$. B. Mixed large and small particles. Bar $=10 \mu \mathrm{m}$. C. Particles in the $1 \mu \mathrm{m}$ range resting on the surface of a larger particle. Bar $=1 \mu$. D. Sub-micrometer particles present in the mist. Bar $=1 \mu \mathrm{m}$. E. EDS spectrum indicating the presence of zinc in the particles. F. EDS spectrum indicating the presence of manganese in the particles. 